\title{
Noise-induced polarization switching in complex networks
}

\author{
Jan O. Haerter, ${ }^{1,2}$ Albert Díaz-Guilera, ${ }^{1,3}$ and M. Ángeles Serrano ${ }^{1,3,4}$ \\ ${ }^{1}$ Departament de Física de la Matèria Condensada, Universitat de Barcelona, Martí i Franquès 1, 08028 Barcelona, Spain \\ ${ }^{2}$ Niels Bohr Institutet, Kфbenhavns Universitet, Blegdamsvej 17, 2100 Kфbenhavn Ø, Denmark \\ ${ }^{3}$ Universitat de Barcelona Institute of Complex Systems (UBICS), Universitat de Barcelona, Barcelona, Spain \\ ${ }^{4}$ ICREA, Pg. Lluís Companys 23, 08010 Barcelona, Spain
}

(Received 2 August 2016; revised manuscript received 9 February 2017; published 11 April 2017)

\begin{abstract}
The combination of bistability and noise is ubiquitous in complex systems, from biology to social interactions, and has important implications for their functioning and resilience. Here we use a simple three-state dynamical process, in which nodes go from one pole to another through an intermediate state, to show that noise can induce polarization switching in bistable systems if dynamical correlations are significant. In large, fully connected networks, where dynamical correlations can be neglected, increasing noise yields a collapse of bistability to an unpolarized configuration where the three possible states of the nodes are equally likely. In contrast, increased noise induces abrupt and irreversible polarization switching in sparsely connected networks. In multiplexes, where each layer can have a different polarization tendency, one layer is dominant and progressively imposes its polarization state on the other, offsetting or promoting the ability of noise to switch its polarization. Overall, we show that the interplay of noise and dynamical correlations can yield discontinuous transitions between extremes, which cannot be explained by a simple mean-field description.
\end{abstract}

DOI: 10.1103/PhysRevE.95.042305

\section{INTRODUCTION}

Bistable behavior in systems which can operate in two competing modes is common in a wide range of domains, from cell biology [1,2] to social dilemmas [3]. Concurrently, stochastic fluctuations are inherent to most of these systems $[4,5]$. In general, many of such systems can be described as a collection of constituents which interact with one another. Yet any constituent will be strongly limited in the number of its interaction partners. Take social networks, where it is well known that cognitive abilities limit the number of stable connections available to any individual [6]. In this work, we address a model capturing bistability, the presence of noise, and finite connectivity.

Bistability can loosely be seen as the existence of a "probability barrier" between two preferred states. In bistable systems of finite size, this barrier can be overcome, and an obvious effect of noise is to cause fluctuating transitions between the preferred states. However, noise may also impact on bistability intrinsically by unfolding a bistable phase into a monostable one, hence effectively removing the barrier altogether. This effect has been proved in very different systems, like autocatalytic chemical reactions [7] and decision making in financial markets [8]. This effect can be observed even when dynamical correlations between constituents are not significant, like in the Ising model on interconnected complex networks [9]. Heterogeneity and nontrivial topological features in the patterns of interaction can further impact the dynamics [10-13].

Even in systems without bistability, noise can induce nontrivial phenomena [14,15]. A paradigmatic example is the Brownian ratchet, where directed motion can be induced by combining Brownian motion with an asymmetric potential [16]. A discretized version of this, the "Parrondo paradox," describes the alternation of two processes-each with losing expectations - which combine to produce a winning result [17-20]. At the base of the Parrondo paradox is an intrinsic asymmetry of transition probabilities; increases of a variable obey different rules than decreases. Such asymmetry leads to some states becoming more likely than others. Incorporating (unbiased) noise then acts to nudge the variable to overcome low probability transitions.

Here we consider the joint effect of dynamical correlations and noise on bistability. We study a system that is bistable in the mean field, i.e., when dynamical correlations are absent. In the mean-field system, bistability is lost for a sufficient level of noise at which the system acquires a neutral mean state. When dynamical correlations become significant, and abrupt irreversible switching from bistability to polarized monostability is found, the neutral mean state is not accessible. We consider constituents to be organized on a network structure, enabling interaction patterns ranging from the mean field (infinite fully connected network) to strong correlation (sparsely connected network). We derive an analytic expression for the fixed points of the mean-field dynamics and a tree approximation for regular random graphs, which is solved numerically. Scale-free, Watts-Strogatz, and multiplex networks are also investigated using numerical simulations.

The structure of this article is as follows: In Sec. II we first define the model. Within this model, we then discuss fully connected systems (Sec. III), for both finite and infinite system size. For sparse connectivity, we analyze the effect of dynamical correlations between nodes and explore several ways in which these can lead to switching of polarization (Secs. IV and V). We further study the case where two networks are coupled by a copying process, allowing one system to influence the state of the other (Sec. VI) and conclude (Sec. VII).

\section{MODEL}

To model bistability, we use a simple three-state dynamical process $[2,21,22]$ in which nodes go from one pole to another 
(a)

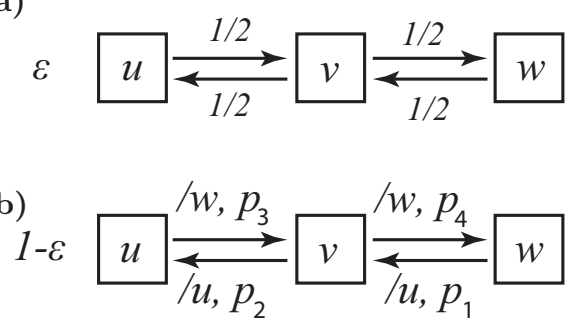

FIG. 1. Three-state model for bistability. (a) Unconditional random process. With probability $\varepsilon$, a node is selected and changes its state with probability $1 / 2$ as shown. (b) Conditional random process. At probability $1-\varepsilon$, transitions are conditional on the state of a selected neighbor, e.g., transition $u \rightarrow v$ for a given node is possible with probability $p_{3}$ if the selected neighbor is in $w$, and no transition takes place otherwise.

through an intermediate state, and we combine it with noise affecting all constituents. We consider undirected networks of $N$ nodes, where each node can be in one of three states, labeled $u, v$, and $w$ (Fig. 1). In the following, for simplicity of presentation, we use these three symbols both for the state label and for the state density. These states could encode unmethylated, hemi-methylated, and fully methylated $\mathrm{CpG}$ sites in DNA methylation, or left-wing, undecided, and rightwing orientation in politics. Transitions between the extreme states $u$ and $w$ always visit the intermediate state $v$ and can occur in two ways: type-1 transitions are unconditionally random and depend only on the state of the affected node [Fig. 1(a)]. This is analog to Brownian motion, where nodes perform random walks in the space of configurations. Type-2 transitions are conditionally random, as they depend on the state of neighbors [Fig. 1(b)]. The dynamics proceeds as follows: a node $i$, termed the "target node," is chosen at random. With probability $\varepsilon$, respectively, $1-\varepsilon$, a type- 1 or type- 2 transition is chosen. If type- 1 is chosen, any of the possible transitions for $i$ is selected at equal probability $1 / 2$. That is, if $i$ is in state $u, i$ will transition to the state $v$ at probability $1 / 2$, and otherwise remain in $u$. If $i$ is in state $v$, it has equal probability $1 / 2$ to transition to either $u$ or $w$. If type- 2 is chosen, one of $i$ 's neighbors $j$, termed the "partner," is first selected at random. Depending on the state of $j$, a transition will occur at $i$ with the corresponding probability $p_{l}, l \in\{1, \ldots, 4\}$ [Fig. 1(b)]. That is, if $i$ is in state $u$ and $j$ in $w$, the probability for $i$ to transition to $v$ is $p_{3}$, and for other states of $j$ the transition probability is zero. Note that only four of the nine combinations of states of $i$ and $j$ lead to conditional transitions; others are not affected.

\section{INFINITE FULLY CONNECTED SYSTEMS}

Dynamical correlations between the states of any two nodes in fully-connected networks with $N \rightarrow \infty$ become negligible. See Appendix A for the analytical treatment of the cases $N=2$ and $N=3$. In the infinite system limit, the probability for one node to be influenced repeatedly by any given neighbor in two consecutive updates decays as $1 / N$. For sufficiently large $N$ it becomes appropriate to neglect all correlations and assume that any node only "feels," i.e., responds to, the mean densities $\bar{u}, \bar{v}$, and $\bar{w}$. With $\bar{u}+\bar{v}+\bar{w}=1$, one is left with two coupled nonlinear differential equations describing $d \bar{u} / d t$ and $d \bar{w} / d t$.

We now show that these equations allow for two stable fixed points. In the following, we let $p_{1}=p_{4}=1$ and $p_{2}=p_{3} \equiv p$, leaving only $p \leqslant 1$ free. We use $\bar{q}$ for the system average of any quantity $q$ as well as the symbol $M \equiv w-u$ to denote net polarization or "magnetization." With these values, the two stable fixed points are such that $\bar{M} \neq 0$ when $\varepsilon<\varepsilon_{c}(p)$. For $\varepsilon \geqslant \varepsilon_{c}(p)$, a stable fixed point exists at $\bar{M}=0$. In the mean field, the conditional probabilities in Fig. 1(b) can be described as contingent on mean densities of the three states $\bar{u}, \bar{v}$, and $\bar{w}$. In the following expressions we drop the overbar for simplified notation. The resulting dynamical equations then read

$$
\begin{aligned}
& \dot{u}=f(u, w), \\
& \dot{w}=g(u, w) .
\end{aligned}
$$

The functions $f(u, w)$ and $g(u, w)$ depend only on the densities of the states $u$ and $w$ because the density of $v$ results from the conservation of probability, i.e., $v(w, u)=1-w-u$. Using the conditions in Fig. 1, the expressions on the RHS of Eqs. (1) and (2) are

$$
\begin{gathered}
f(u, w)=(1-\varepsilon) p u(1-2 w-u)+\varepsilon(1-w-2 u), \\
g(u, w)=(1-\varepsilon) w(1-2 u-w)+\varepsilon(1-2 w-u) .
\end{gathered}
$$

For these simplified equations, there is a line of (neutral) fixed points at $u=w=1 / 3$ for all $\varepsilon$. Linear stability analysis of these fixed points reveals a bifurcation point at

$$
\varepsilon_{c}=(1+3 / \sqrt{p})^{-1} .
$$

For $\varepsilon<\varepsilon_{c}$ any neutral fixed point is an unstable saddle, while for $\varepsilon>\varepsilon_{c}$ it is a stable node. The characterization of the remaining fixed points depends on $p$.

For $p=1$, in the diagram of $M=w-u$ [see gray curves in Fig. 2(a)], two symmetric branches of stable fixed points exist for $\varepsilon<\varepsilon_{c}=1 / 4$, which approach \pm 1 as $\varepsilon \rightarrow 0$. In this case, solving for $f(u, w)=g(u, w)=0$ yields the fixed points

$$
\begin{aligned}
& u=\frac{1}{2}\left(1-\varepsilon^{\prime} \pm \sqrt{1-2 \varepsilon^{\prime}-3 \varepsilon^{\prime 2}}\right), \\
& w=\frac{1}{2}\left(1-\varepsilon^{\prime} \mp \sqrt{1-2 \varepsilon^{\prime}-3 \varepsilon^{\prime 2}}\right),
\end{aligned}
$$

where we have defined $\varepsilon^{\prime} \equiv \varepsilon /(1-\varepsilon)$ for simplified notation. The difference $w-u$ becomes

$$
M=w-u= \pm \sqrt{1-2 \varepsilon^{\prime}-3 \varepsilon^{\prime 2}} .
$$

For $p<1, \varepsilon_{c}$ diminishes and the branches of stable fixed points become asymmetric [gray curves in Fig. 2(b)]. An additional line of unstable fixed points appears, which joins the bifurcation point and the upper branch of stable fixed points. In the regime of low noise, i.e., $\varepsilon \rightarrow 0$, the system approaches one of the stable fixed points $w-u= \pm 1$. As the noise level is increased, the state of the system follows the corresponding branch of stable fixed points, until it eventually reaches $w-u=0$. In the case of the lower branch, i.e., $w-u<0$, the approach is continuous. In the case of the upper branch, i.e., $w-u>0$, there is a jump discontinuity [compare Fig. 2(b)]. However, in all cases, the neutral value 

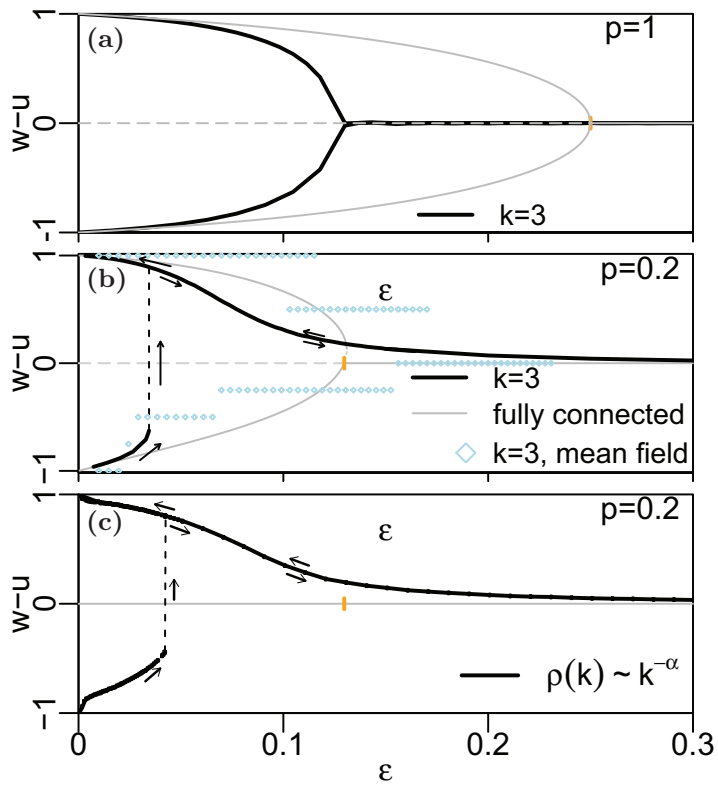

FIG. 2. Polarization as a function of noise. (a) Symmetric process, $p=1$, on a degree regular random graph with $k=3$ (bold black lines) when initializing in the two extremal states $\bar{w}=1$ and $\bar{u}=1$, respectively, and infinite, fully connected network (gray curves). Solid (dashed) gray lines show stable (unstable) fixed points; vertical orange mark indicates the bifurcation point $\varepsilon_{c}(p)=(1+3 / \sqrt{p})^{-1}=1 / 4$ for the fully connected network. (b) Similar to (a) but for $p=0.2$, i.e., for a nonsymmetric dynamics. Dashed vertical line marks the abrupt transition from $\bar{M}<0$ to $\bar{M}>0$. The vertical orange line marks $\varepsilon_{c}(0.2) \approx 0.13$. Light blue symbols mark the distribution maxima obtained through the tree approximation (Fig. 6) for $k=3$. Arrows mark the path taken by $\bar{M}$ when starting from $\bar{M}=-1$ and $\varepsilon=0$, then first increasing $\varepsilon$ towards unity and then again reducing $\varepsilon$ to zero. (c) Similar to (b) but for scale-free degree distribution with $\alpha=2.4$ and $\langle k\rangle \approx 3$. Hysteresis in (c) is similar as in (b), arrows not shown. System size for simulations: $N=5000$ (see Appendix B), in (b) near the transition: $N=20000$.

of $w-u=0$ is approached monotonically as the noise level is increased. The effect of noise, hence, is to reduce the polarization of the system state and eventually yield a configuration where the three possible states of the nodes $u, v$ and $w$ are equally likely.

\section{SPARSELY CONNECTED SYSTEMS}

How does noise influence bistability in the presence of dynamical correlations? To answer this, we explore networks where dynamical correlations are important, hence connectivity is low $(k \ll N)$, but system size is large $(N \gg 1)$. We first simulated graphs where each node has identical degree $k$. Dynamical correlations are visually reflected in the phase portrait (Fig. 3) where trajectories are distorted compared to the fully connected system (Fig. 3) and often cross themselves or one another.

For $p=1$, which makes the model symmetric regarding the states $u$ and $w$, we yield a symmetric bifurcation diagram when initializing the system in the extremal states $\bar{M}= \pm 1$, as expected. This diagram is qualitatively similar to the

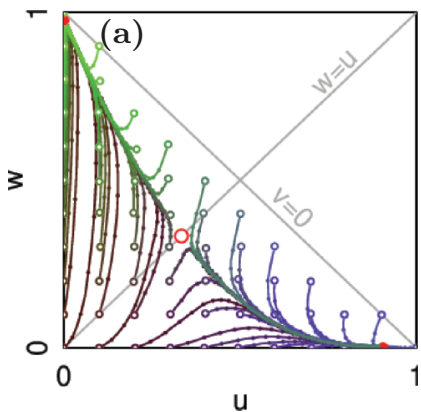

u

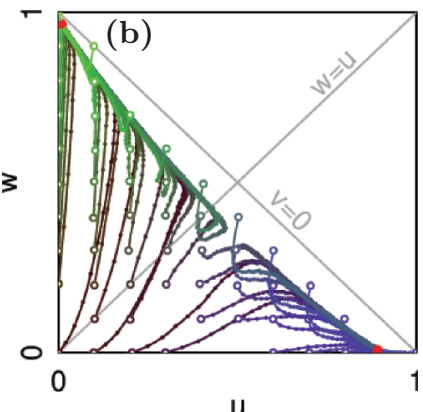

FIG. 3. Phase portraits for fully and sparsely connected systems. (a) Fully connected system with $p=0.2$ and $\varepsilon=0.02$, i.e., in the bistable phase. Gray open, red filled, and red open circles represent initial conditions, stable fixed points, and unstable fixed points, respectively. Colors of curves distinguish trajectories. (b) Similar to (a) but for a degree-regular random graph with $\langle k\rangle=3$. Panels were obtained using simulations with 30000 nodes.

one obtained for the fully connected system. However, the bifurcation point in the network, referred to as $\varepsilon^{*}(p)$, has now moved to considerably lower values of $\varepsilon^{*}(p=1) \approx 0.13<$ $\varepsilon_{c}(p=1)$ [Fig. 2(a)], an effect of the dynamical correlations retained by demanding small $\langle k\rangle$. Bistability has become more sensitive to noise.

Second, we introduce asymmetry $(0 \leqslant p<1)$, which means that conditional transitions (i.e., contingent on either $u$ or $w$ at neighboring sites) back and forth between $u$ and $v$ are now less frequent than conditional transitions between $v$ and $w$. This changes the picture qualitatively [Fig. 2(b)] as compared to the symmetric case: while the system remains bistable at low noise $(\varepsilon<.04, p=0.2)$, the lower branch of fixed points is more sensitive to $\varepsilon$ and collapses when $\varepsilon$ is increased. The system switches abruptly-i.e., changes the sign of $M$-to a $w$-dominant configuration, and remains polarized there, even as noise is increased further. To verify that the transition is abrupt, i.e., first order, we simulated the corresponding double well $-\ln [\rho(M)]$ where $\rho(M)$ is the probability density function of $M$ and checked that the potential well at negative $M$ disappears without merging with that at $M>0$; see Fig. 12 .

Reducing $\varepsilon$ again below $\varepsilon^{*}(p)$ does not restore the $u$ dominant state, the system remains polarized at $\bar{M}>0$. Only when $\varepsilon \rightarrow 1$ does the system approach the neutral state where $\bar{M}=0$. Henceforth, we use the terms "vulnerable" and "resilient" for the corresponding branches of the system. The "vulnerable" branch is the one that collapses abruptly when noise is increased, while the "resilient" branch remains in its overall polarization, albeit with a gradual reduction in magnitude.

Third, we ask whether such abrupt switches also occur in heterogeneous graphs. We synthesized random networks with scale-free degree distributions $P(k) \propto k^{-\alpha}, \alpha \approx 2.4$, which gave similar $\langle k\rangle \approx 3$ as in the previous simulation for the degree regular network. The qualitative features are preserved [Fig. 2(c)]: bistability is present at low noise, but an abrupt switch from $\bar{M}<0$ to $\bar{M}>0$ occurs at a value of noise significantly lower than that of the bifurcation in the fully connected graph for comparable $p$. Heterogeneous degrees 


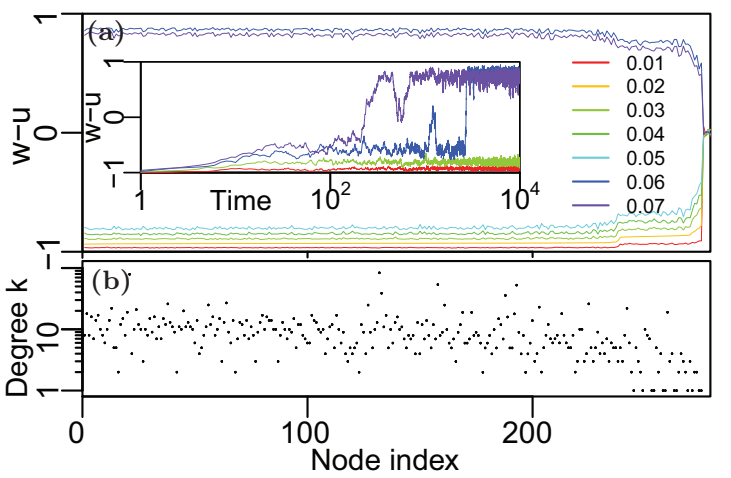

FIG. 4. Dynamics on the nervous system of C. elegans. Electric and chemical synaptic connections [23] (279 nodes). (a) Timeaveraged values of $M$ for individual nodes after completion of the transient. Nodes were sorted by increasing $M$ for the simulation of $\varepsilon=0.02$ (orange curve, see legend). This ranking was maintained when plotting the results for other values of $\varepsilon$. Colors (red to purple) correspond to increasing values of $\varepsilon \in\{0.01, \ldots, 0.07\}$. Inset: Several representative time series of network average $\bar{M} \equiv \bar{w}-\bar{u}$ when initializing all nodes at $M=-1$ at $t=0$. Time is measured in units of system updates. (b) Black points indicate the degree of each node. Note the logarithmic vertical axis. The nodes to the very right have degree zero and are therefore not shown on the log scale. Note that degree-zero nodes have no interactions with other nodes, therefore they are not bistable ( $w-u \approx 0$ in panel a for these nodes).

imply that the expected state of any individual node may also be different from that of any other. Indeed, states not only depend on a node's degree, but additionally on the broader context nodes play within the network. To illustrate this, we run the dynamical process on the nervous system of $C$. elegans [23] (Fig. 4). When extracting any node's stationary temporal mean state, we find that a node's neighborhood defines its rank, i.e., relative mean state within the network, and this rank is roughly unchanged, even when varying noise or initial conditions. Further, random graphs with Poissonian degree distributions as well as the two-dimensional square lattice (Fig. 5) again gave qualitatively similar behavior as in Figs. 2(b) and 2(c).

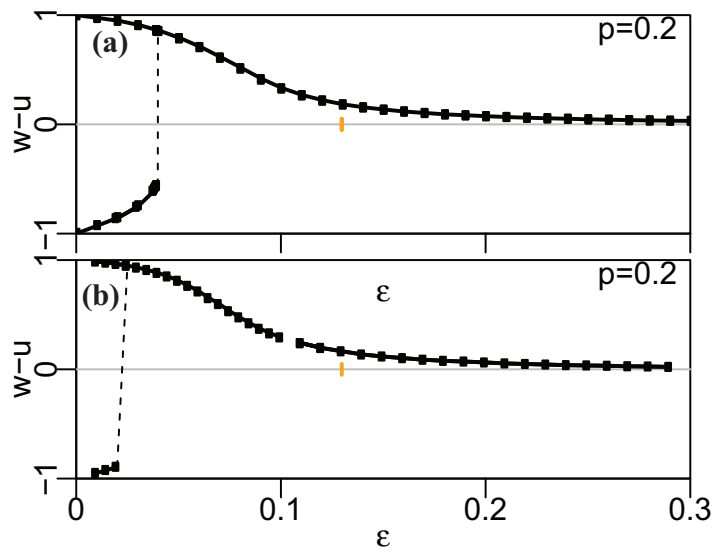

FIG. 5. Transitions for additional network structures. Similar to Fig. 2(c) $(N=5000)$ but for a Poissonian degree distribution with $\langle k\rangle=3$ (shown in a) as well as a square lattice (shown in b).

\section{CAUSE OF POLARIZATION SWITCHING-ANALYTICAL APPROXIMATION}

To explore further the origin of the polarization switching we developed an analytical approximation for sparsely connected networks. We focus on a sparse degree regular network, i.e., $k \ll N$. The discussion on the $N=2, N=3$ (see Appendix A), and fully connected systems highlights that any mean-field approach ignoring dynamical correlations is bound to fail. Further, any approximation should incorporate states formed by more than two nodes, given that two nodes do not show any change of sign in polarization whereas systems with more than two nodes do.

We consider that a node and its $k$ nearest neighbors form a cluster of $k+1$ nodes and $k$ links, and use a tree approximation, in which any two nodes are connected only by one path; see Fig. 6. In general, the configuration space of node states for this cluster spans $s_{k} \equiv 3^{k+1}$ different configurations. We refer to any of the cluster states $i$ as $\chi_{i}$ and to the probability of this state being occupied as $\Pi_{i}$. The steady-state solutions could be computed by writing the dynamical equations for the transition probabilities between these states.

However, this would ignore all interaction with the surroundings of the cluster. In order to achieve a better approximation, consider the following: When all nodes have equal connectivity, choosing a target node and its partner is equivalent to choosing a link and assigning one of the two nodes involved as the target node. Now consider all links within the cluster (shown in red in Fig. 6) as well as those outside the cluster (shown in black). There are $k$ ways to choose an internal link, and the target node will always lie within the cluster. There are $k(k-1)$ ways to choose external links; however, the target node will only lie within the cluster at probability $1 / 2$. Together, the probability of an internal updating is then

$$
p_{\text {int }}=1-p_{\text {ext }}=\frac{k}{k+k(k-1) / 2}=\frac{2}{1+k} .
$$

We now need to evaluate separately the selection of internal and external links. Selecting an internal link and target node generates a transition matrix for all cluster states. A given state $\chi_{i}$ thereby has a probability $P\left(\chi_{i} \rightarrow \chi_{j}\right)$ to transition to state $\chi_{j}$. This probability is straightforward to determine, given the schematic in Fig. 1 and noting that any possible transition

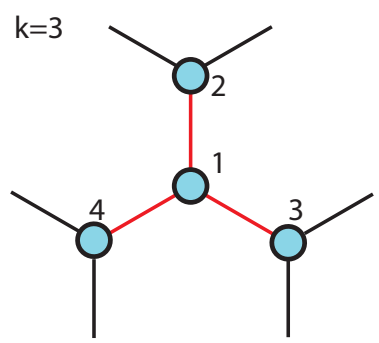

\begin{tabular}{cccc}
1 & 2 & 3 & 4 \\
\hline$u$ & $u$ & $u$ & $u$ \\
$u$ & $u$ & $u$ & $v$ \\
$u$ & $u$ & $u$ & $m$ \\
$u$ & $u$ & $v$ & $v$ \\
$u$ & $u$ & $v$ & $m$ \\
$u$ & $u$ & $m$ & $m$ \\
. &. &. &. \\
. &. &. &.
\end{tabular}

FIG. 6. Tree approximation. Example for connectivity $k=3$. Bethe lattice where a central node is connected to three nearest neighbors, which themselves are each connected to three nearest neighbors. We distinguish internal and external links, which are indicated by red and black, respectively. Also shown are several configurations of unique four-node states, i.e., those that do not map onto one another by permutations of the individual single-node states. 
is constrained to states that differ only by one elementary move. For example, the transition $\{u u u v\} \rightarrow\{u u u w\}$ is possible when node four is selected as the target site and its probability is then $\varepsilon / 2$. Conversely, the transition probability $P(\{u u u v\} \rightarrow\{u u u u\})=(1-\varepsilon) p+\varepsilon / 2$ since a coordinated move is now possible.

Updating an external link can modify any but the central node of the cluster. We now need to consider the updating of external cluster states, i.e., clusters with central nodes located at sites $2, \ldots, k+1$ w.r.t. the original cluster. These updates require some bookkeeping: for a given cluster state $\chi_{i}$ not all external cluster states will be possible. A sum needs to be carried out over nodes $j \in\{2, \ldots, k+1\}$, where the respective states of the pairs $(1, j)$ constrain the possible external cluster states. Given this subset of states, all possible transitions are again enumerated. It is then back-tracked how these transitions affect the respective node $j$, which in turn leads to an update of the internal cluster state.

The temporal evolution, in time units of $N$ node updates, of the occupation probability $\Pi_{i}$ hence is

$$
\begin{aligned}
\dot{\Pi}_{i}= & p_{\text {int }}\left[\sum_{j=1}^{n} \Pi_{j} P\left(\chi_{j} \rightarrow \chi_{i}\right)-\Pi_{i} P\left(\chi_{i} \rightarrow \chi_{j}\right)\right] \\
& +\left(1-p_{\text {int }}\right) \sum_{x=2}^{k+1}\left[\sum_{j} \Pi_{j} P\left(\left\{s_{q}\right\} \mid s_{1}, s_{x}^{\prime}\right) P\left(\chi_{s_{1}, s_{x}^{\prime},\{q\}} \rightarrow \chi_{s_{1}, s_{x},\{q\}}\right)-\Pi_{i} P\left(\left\{s_{q}\right\} \mid s_{1}, s_{x}\right) P\left(\chi_{s_{1}, s_{x},\{q\}} \rightarrow \chi_{s_{1}, s_{x}^{\prime},\{q\}}\right)\right] .
\end{aligned}
$$

Here

$$
P\left(\left\{s_{q}\right\} \mid s_{1}, s_{x}\right) \equiv \frac{\Pi_{1, x,\{q\}}}{\sum_{\{q\}} \Pi_{1, x,\{q\}}}
$$

is the conditional probability that the remaining nodes $\{q\}$ are in the states $\left\{s_{q}\right\}$ given that nodes 1 and $x$ are in the single-node state $s_{1}$ and $s_{x}$, respectively. The sum in the denominator extends over all configurations of external states that are compatible with the single node states at sites 1 and $x$ and serves as a normalization.

Notably, all configurations $\chi_{i}$ of the internal cluster can contribute to the first term of Eq. (10), while only those configurations $\Pi_{j}$ which are compatible with a given central node state $s_{1}$ and a state $s_{x}$ at a given peripheral node contribute in the second term. Note further that in the first term only linear contributions in the occupation probabilities $\Pi_{i}$ occur, while the second term involves also product terms of probabilities $\Pi_{i}$.

The approximation now consists in the closure of correlations up to the size of the cluster. It is implicitly assumed that longer-range correlations do not contribute and can be neglected.

Equation (10) constitutes a set of nonlinear equations in the occupation probabilities $\Pi_{i}$ and has to be solved numerically. The density of states is initially peaked at low and high values of $w-u$, with the high- $u$ state the most likely. As noise is increased, one peak gradually disappears and a single-peak distribution results. The crucial features of the density of states can be represented more compactly by extracting the peaks of the distribution function (Fig. 13). For the cluster consisting of four sites, excluding the surroundings, Fig. 13(a) shows the dependence of maxima on the noise $\varepsilon$. Indeed, the distribution is bimodal at low $\varepsilon$, whereas for larger noise $(\varepsilon>0.15)$ the distribution is unimodal. We compare this to the solution where also the surroundings are taken into account [Fig. 13(b)]. Now the peaks for the $u$-dominant state decay more rapidly, and the pattern is much more skewed than in Fig. 13(a). At a value of $\varepsilon \approx 0.04$ the distribution is unimodal, and peaks are present for the $w$-dominant state. This result should be compared to the simulation result in Fig. 2(b), where similar qualitative features are present.

Importance of clustering-We note that the mean-field calculation based on the star motif assumes a tree-like topology and hence disregards effects of clustering and long-range connections. To distinguish those effects, we performed additional analysis by implementing Watts-Strogatz networks [24] to interpolate between a regular ring lattice and a random graph. As a result of repeated rewiring [Fig. 7(a)], which gradually reduces clustering by increasing long-range connectivity, the switching $\varepsilon^{*}(p)$ systematically moves towards larger values of noise [Fig. 7(b)]. We see this as an evidence of the role of long-range connections in enabling the presence of two stable states at small values of noise. Bistability can be suppressed by increased locality with associated node-node dynamical correlations.

This interpretation further implies the existence of dynamical correlations reaching beyond the immediate neighborhood of a given node. This is again in line with the results from the neural wiring diagram of C. elegans [23] (Fig. 4), where we consider the long-term average of each individual network node and find that a node's degree alone is not a sufficient predictor of its average state within the network [compare Figs. 4(a) and 4(b)]. Remarkably, even when changing $\varepsilon$, nodes maintain their relative polarization w.r.t. the remaining network; i.e., the curves shown in Fig. 4 are approximately still smooth when noise is varied.

\section{TWO-LAYER MULTIPLEX NETWORKS}

Finally, we form a multiplex network by combining two layers $\mathrm{A}$ and $\mathrm{B}$, where nodes can change their state by influences from either of the layers and the layers have distinct model parameters. For simplicity, we consider each layer to consist of statistically equivalent regular random graphs. We allow the dynamics in layer $A$ to be defined by the parameters used previously (i.e., $p_{1}=p_{4}=1, p_{2}=p_{3}=0.2$ ), where $\bar{M}>0$ gives its resilient branch. When uncoupled, layer A would hence behave as described above. In layer B, rates 

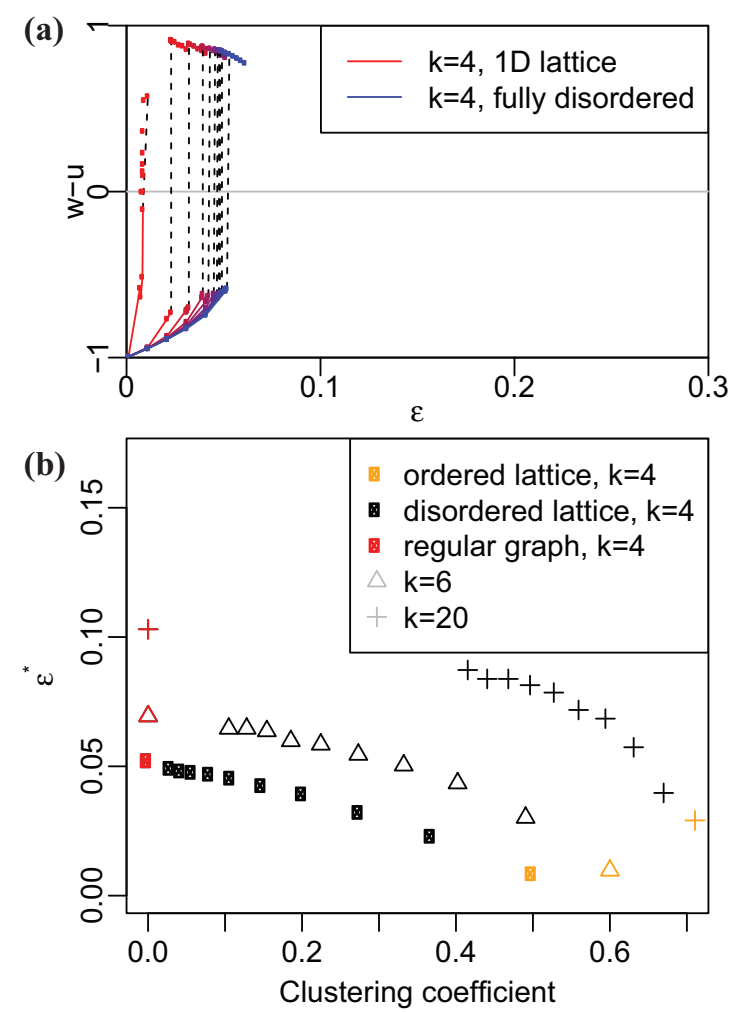

FIG. 7. Effect of clustering. (a) Transitions for varying levels of disorder for $k=4, \varepsilon^{*}$ increases with disorder. The fully disordered system is a regular graph of degree $k=4$. System size: $N=2000$. (b) Summary of $\varepsilon^{*}$ for the simulations of varying connectivity ( $k=$ $4,6,20)$ as a function of clustering coefficient.

are taken to be smaller: $p_{1}=p_{4}=0.1, p_{2}=p_{3}=0.5$ and swapped regarding $u$ and $w$. Hence, B's resilient branch is characterized by $\bar{M}<0$ [Fig. 8(a), inset]. Both subsystems are initialized as $M=1$ and allowed to reach a steady state. We couple A and B by requiring a randomly chosen fraction $c_{f}$ of nodes to have shared states in the two layers, i.e., their state in one network is copied to the other network at any update. The remaining fraction of nodes never transfer their states from one layer to the other. At every time step, A or $\mathrm{B}$ is chosen at equal probability, and a random node of that network is updated as before.

At low noise $\varepsilon$, both systems are $w$-dominant (Fig. 8). As $\varepsilon$ is increased, the behavior depends on $c_{f}$. For weak $c_{f}$, B will eventually transition to its resilient configuration, where $M<0$. Notably, this transition is still abrupt for small $c_{f}$ [Fig. 8(a), red curves]. As $c_{f}$ is moderately increased (purple curves), the abrupt switch of B is first reduced and eventually disappears, but B still shows changing sign of $M$. The associated reduction of jump size appears to be continuous in the sense that the magnitude of the jump occurring at the zero crossing is gradually reduced as $c_{f}$ is increased. Conversely, layer $\mathrm{A}$ is also impacted upon by $\mathrm{B}$, since the magnitude of $\bar{M}$ in $\mathrm{A}$ is somewhat reduced by the coupling. As $c_{f}$ is increased further, even the change of sign finally disappears (blue curves). For sufficiently large coupling (e.g., $c_{f}=0.64$ ) the curves of $\mathrm{A}$ and $\mathrm{B}$ assimilate and the magnitude of $\bar{M}$ at low noise becomes maximal: the copying process between layers
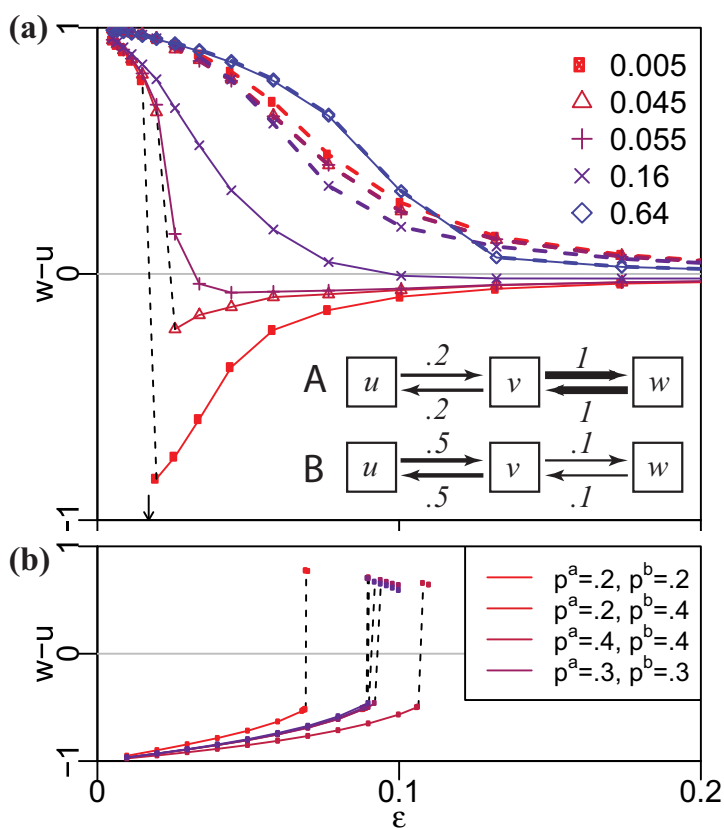

FIG. 8. Dynamics in a two-layer multiplex with variable coupling. (a) Both layers A (dashed lines) and B (solid lines) are initialized at $M=1$ for different values of $\varepsilon$ (see schematic, inset). The plot legend (top right) indicates various values of coupling $c_{f}$ increasing from 0.005 to 0.64 for colors ranging from red to blue. Vertical arrow indicates the approximate position of $\varepsilon^{*}$ for the isolated system B. $N=2000$. (b) Transition for perfect coupling and various combinations of model parameters. Parameters $p^{A}$ and $p^{B}$ correspond to the parameter $p$ in the model (Fig. 1) for the two-layer networks $A$ and $B$. Updating a node in one subsystem will also update the node in the other subsystem. Curves obtained for subsystems of $N=2000$ nodes each and degree $k=3$. Mixing two layers has the main effect of averaging the rates of the two subsystems, seen by comparing the cases of $p^{a}=0.2, p^{b}=0.4$ to $p^{a}=0.3, p^{b}=0.3$.

is now so efficient that they act as a single layer with enhanced effective degree $\tilde{k}>3$ and intermediate effective parameters [see Fig. 8(b)]. Hence, the effect of combining layers is to dilute the correlations between any two neighbors by the larger number of influences from their enlarged neighborhood. The relative frequencies of updating (e.g., the rates $p_{l}$ ) in the two layers determine which of the two will be able to impose its state on the other.

We explored variants of this setup (Fig. 9). When assuming that now all nodes are coupled between the two layers $\left(c_{f}=1\right)$, but copying of states proceeds at a certain probability $c_{r}<1$ at every move, the dynamics remains qualitatively similar but changes quantitatively [Fig. 9(a)]. To determine whether $c_{r}$ or $c_{f}$ is more efficient in destabilizing B's resilient state, we fix the product $c_{r} c_{f}$ and then vary the two rates simultaneously [Fig. 9(b)]. This analysis shows that large $c_{f}$ is able to pull layer B through the switch, i.e., fixed coupling is preferable in destroying a coherent configuration of $\mathrm{B}$ by the coupling to $\mathrm{A}$. We further explored the case where systems A and B have similar setup but vary in the strength of $p$ [Fig. 9(c)]. For uncoupled $\mathrm{A}$ and $\mathrm{B}$, both initialized in the vulnerable state, the system of smaller $p$ will transition at lower $\varepsilon$. When coupling 


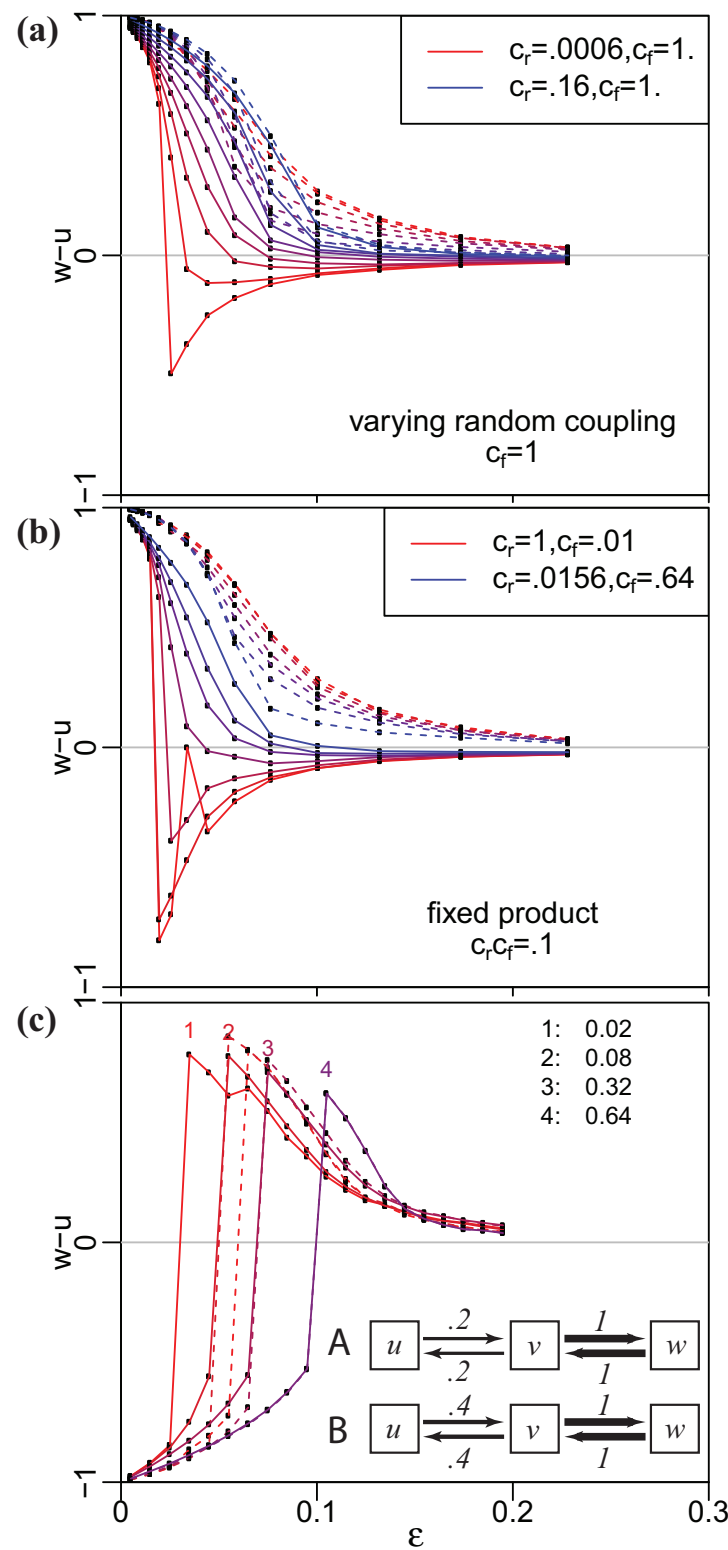

FIG. 9. Dynamics in a two-layer multiplex with alternative couplings. Dashed (solid) lines represent systems A and B, respectively. (a) Similar to Fig. 8 but keeping $c_{f}=1$ maximal, i.e., all nodes are capable of copying their states. Now $c_{r}$ is varied as shown in the legend. (b) Similar to Fig. 8 but keeping the product $c_{r} c_{f}=0.1$ fixed. Parameters of different curves as shown in the legend. (c) Coupling two systems as shown in inset. $c_{r}=1$. Numbers near lines and in the legend represent the fraction of nodes permanently coupled $\left(c_{f}\right)$. System sizes in all panels: $N=2000$.

A and B, the systems will eventually transition at the same value of $\varepsilon$ but the jump size remains different for the two.

\section{CONCLUSIONS}

Elementary interactions in biology or social systems are often between two entities: Within DNA, contacts between one genomal region and another may lead to reactions, e.g., in epigenetics [1,21]. In recent work it has been shown that the dynamics in such systems can often lead to bistable system states, where constituents jointly reach a polarized state, e.g., fully methylated or unmethylated configurations in DNA methylation [2,22]. In social networks, one individual may influence another's opinion. Classical models, e.g., the Voter-type dynamics [25], eventually drift towards a unique absorbing state, which is set by the system parameters. We here propose that a simple modification of the Voter model to a three-state model can already give rise to bistable opinion dynamics: given overall "resetting" of the general opinion to one of the extremal states, a large system will maintain that state indefinitely. Smaller opinion networks may transition stochastically between the two extremal states.

Notably, when connectivity is extensive, the system's dynamics could be described within the mean field. However, connectivity is often limited: in cell biology, the range of interaction may be set by the protein superstructure formed by the DNA, limiting available interaction partners. In social networks, it is well known that the number of stable ties a mammalian can form in a social network is constrained by its cognitive capacity [6,26,27]. For humans, limits of social connectivity were proposed to be between 100 and 200 $[6,26,27]$, but typical connectivity, e.g., in e-mail communication is generally much lower (below 10) [27]. It hence becomes important to ask which role dynamical correlations between the states of neighboring constituents play in the dynamics and the effects of noise.

We analyzed noise-induced polarization switching caused by dynamical correlations in a simple three-state model for bistability. Our findings may however generalize to other models that induce similar dynamics. The switching occurs only when dynamical correlations are present (low connectivity) and can then be described as a first-order irreversible transition from bistability to monostability. Without dynamical correlations, the switching disappears and noise can induce only a neutral mean state.

Our analysis suggests that this polarization switching exists in the intermediate regime where local dynamical correlations are present and nonlocal coupling allows for bistability to be maintained. Purely local systems, that is, highly clustered networks, do not show signatures of the switching. When two networks interact, the dominant one imposes its resilient state on the other as the coupling increases. This suggests that the propensity of a system to switch polarization under noisy environments can be both counterbalanced or enhanced by its matching with another dominant system.

Conceptually, our findings could be seen as an extreme case of Parrondo's paradox [17-20]. Within this analogy, unbiased noise is a neutral game which transforms a second biased game from overwhelmingly losing to winning when the two are combined. In contrast to Parrondo's paradox, the implications of our findings may be far less subtle: modulating noise could be exploited as a simple mechanism to trigger strong behavioral changes in systems with bistability, e.g., in epigenetics or economics. For several systems coupled within an overall noisy environment, those with higher rates of asymmetric conditional reactions will not only be more resilient but may often dominate other systems that are coupled to them. Our work might allow for a new perspective on a wide range of transitions in noisy bistable systems such as those that are found in biology or the social sciences. 


\section{ACKNOWLEDGMENTS}

J.O.H. thanks Namiko Mitarai and Kim Sneppen for fruitful discussions. M.A.S. acknowledges support from a James S. McDonnell Foundation Scholar Award in Complex Systems; Ministerio de Economia y Competitividad of Spain Projects No. FIS2013-47282-C2-1-P and No. FIS2016-76830C2-2-P (AEI/FEDER, UE); and Generalitat de Catalunya Grant No. 2014SGR608. A.D.-G. acknowledges support from Ministerio de Economia y Competitividad of Spain Projects No. FIS2012-38266-C02-02 and No. FIS2015-71582-C2-2-P (MINECO/FEDER); and Generalitat de Catalunya Grant No. 2014SGR608. J.O.H. acknowledges support by a research grant (13168) from VILLUM FONDEN. All authors acknowledge support from the European Commission FET-Proactive Project MULTIPLEX No. 317532.

\section{APPENDIX A: FULLY CONNECTED FINITE SYSTEMS}

For small systems consisting only of few sites and links connecting each pair of sites, the steady state can be computed exactly. As each node can take one of three states, the $n$-particle state space consists of $3^{n}$ states; i.e., the basis increases exponentially with the number of sites. However, considering permutation symmetry the basis reduces to $m(n)=\left(n^{2}+3 n+2\right) / 2$ states, i.e., the basis then increases only approximately quadratically with the number of sites.

The smallest interacting system is that consisting of only two nodes, connected by a link. Hence, there are $m(2)=6$ basis states. By evaluating all $m(2) \times m(2)$ transition probabilities $P\left(\chi_{i} \rightarrow \chi_{j}\right)$ for transitions between $n$-particle states $\chi_{i}$ and $\chi_{j}$ with $i, j \in\{1, \ldots, m\}$ we write a dynamical equation for the occupation probabilities $\Pi_{i}$ :

$$
\dot{\Pi}_{i}=\sum_{j=1}^{n}\left[\Pi_{j} P\left(\chi_{j} \rightarrow \chi_{i}\right)-\Pi_{i} P\left(\chi_{i} \rightarrow \chi_{j}\right)\right] .
$$

Solving for $\dot{\Pi}_{i}=0$ and considering the vector $\mathbf{M} \equiv$ $\left(M_{1}, \ldots, M_{m}\right)$ of "magnetization," where $M_{i} \equiv w_{i}-u_{i}$ for each $n$-particle state, we obtain the average "magnetization" $\bar{M} \equiv \sum_{i} \Pi_{i} M_{i}$ as a function of noise $\varepsilon$ and the asymmetry parameter $p, \bar{M}=\bar{M}(\varepsilon, p)$.

As a function of $\varepsilon$, for any given value of $p \in[0,1]$ we find that, when $n=2, \bar{M}$ maintains its sign for all values of noise $\varepsilon$ [Fig. 10(a)]. Conversely, for $n=3, \bar{M}$ has a zero crossing as $\varepsilon$ is increased. Retaining only terms linear in $\varepsilon$ we approximate the curve $\bar{M}(\varepsilon, p)$ and, by setting $\bar{M}(\varepsilon, p)=0$, reach an approximate expression for the zero crossing $\varepsilon_{0}(p)$ as a function of $p$ :

$$
\varepsilon_{0}(p)=\frac{p^{2}(1+p)}{2+19 p+24 p^{2}+19 p^{3}+2 p^{4}} .
$$

We find that the function $\varepsilon_{0}(p)$ increases monotonically with $p$ [Fig. 10(b)] and approaches a finite value $\varepsilon_{0}(1)=1 / 33$ as $p \rightarrow 1, p<1$. When $p=1$ the system is symmetric and there is no zero crossing.

Hence, even infinitesimal asymmetry $p \neq 1$ in the model (Fig. 1) can induce a noise-dependent change of sign akin to the original Parrondo paradox. Here, however, the states are the joint states of multiple sites. The effect of noise is to enable
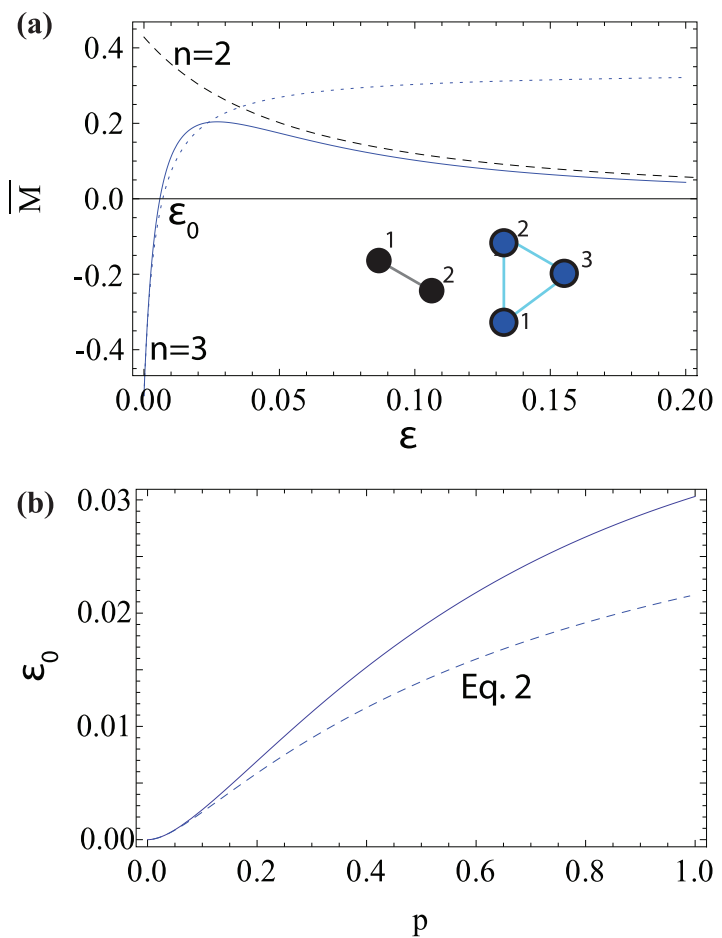

FIG. 10. Finite system. (a) Zero crossing of $\bar{M}$ for $p=0.2$. Dashed black curve shows the analytical solution for $n=2$, where no zero crossing is observed. Dashed blue curve shows the analytical approximation for $n=3$ [Eq. (A2)] (neglecting nonlinear terms in $\varepsilon$ ). Solid blue curve shows the full numerical solution for $n=3$, where all powers of $\varepsilon$ were retained. The zero crossing for $n=3$ is indicated by the label $\varepsilon_{0}$. (b) $n=3$, the level of noise $\varepsilon$ where a zero crossing for $\bar{M}$ occurs for different values of $p$. Dashed and solid blue curves again show analytical approximation [Eq. (A2)] and numerical solution, respectively.

transitions that are unlikely without noise and can eventually drive the system towards another attractor.

Examining larger systems $(n>3)$ numerically, we find the zero crossing and qualitative behavior of $\bar{M}$ to be similar to that of $n=3$. However, when considering a comparison with

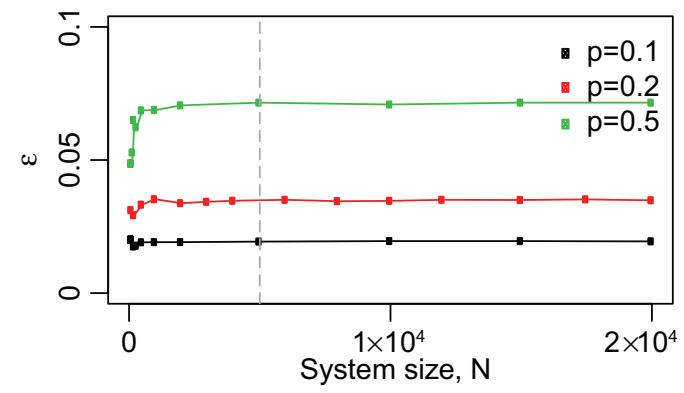

FIG. 11. System size dependence. Simulation for regular graph with $k=3$, but varying system size and $p$. Each curve represents one value of $p$ (marked in legend) and points are simulation results for the transition between $u$-dominant and $w$-dominant states. Lines serve as guide to the eye and connect the points obtained by the simulations. Vertical dashed line indicates $N=5000$, a value we find sufficient for finite size effects to be considered small, unless stated otherwise in the text. 


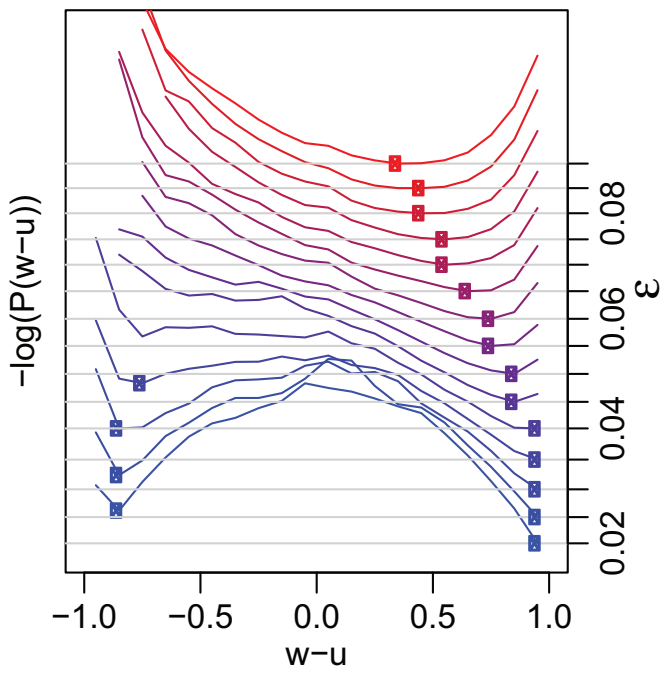

FIG. 12. Potential wells. For each value of noise $\varepsilon$ long simulations with $n=59$ nodes and $k \approx 3 \ll n$ were carried out. As these systems are comparably small, sufficiently many fluctuations between the quasistable states at negative and positive $M$ occur and allow computation of the probability density function $P(M=w-u)$. Curves ranging from blue to red show the function $-\log [P(w-u)]$ for a range of $\varepsilon$ (indicated on the right vertical axis). Inspecting the PDFs shows that the minimum at $M<0$ becomes increasingly weak and finally disappears. As $\varepsilon$ increases, the PDFs obtain an overall slant towards positive $M$. (Details are found in Appendix C.)
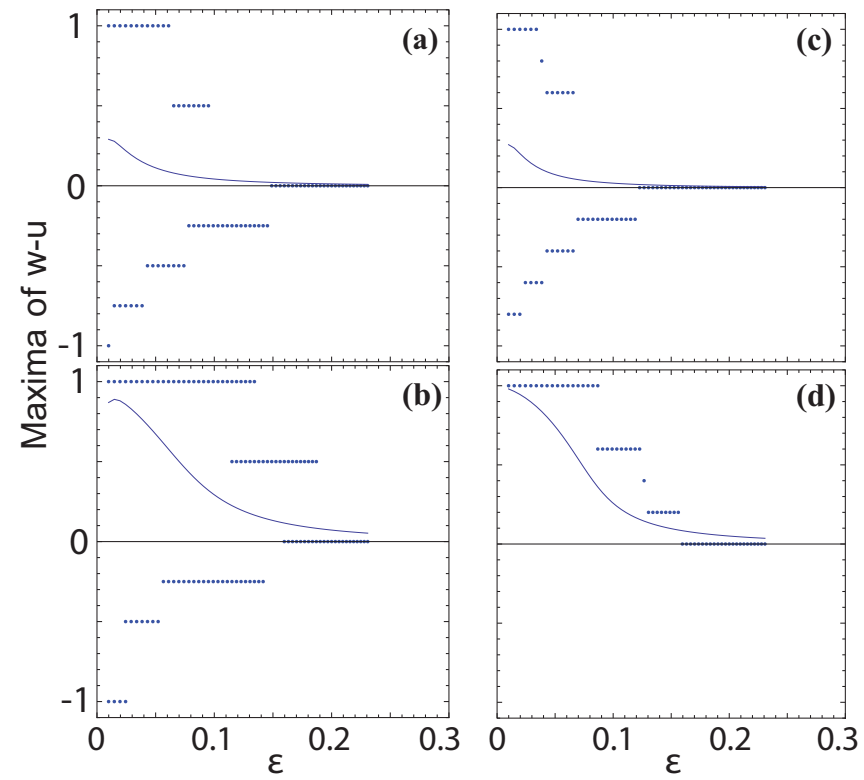

FIG. 13. Maxima of probability distribution function. (a) Star topology consisting of four sites, as shown schematically in Fig. 6 as red links. Solid points show the respective local maxima obtained by inspecting the density of states in the tree approximation (Sec. V). Thin line shows the average value of $w-u$. (b) As in (a) but interacting with surrounding sites, i.e., as in Fig. 6 including the black links. Note the more abrupt decrease of the $u$-dominant state, i.e., maxima for $w$-dominant states persist in noise regions where $u$-dominant states are absent. Note the increase of the average $w-u$ w.r.t. the star topology. (c) Similar to (a) but for a star consisting of five sites. (d) Similar to (c) but for a star consisting of five sites. Note that the maxima at $u>w$ have now disappeared. the noise effect in the Parrondo paradox, as system size is increased, the system at hand will hardly reside in a state near $\bar{M}$. This is because for the model (Fig. 1) bistability is inherent, whereas bistability is absent in the Parrondo paradox.

\section{APPENDIX B: NETWORK SIZE DEPENDENCE OF $\varepsilon^{*}$}

We explored different network sizes to ensure that the system sizes used for our analysis were sufficiently large, and the results reported did not depend on system size (Fig. 11).

\section{APPENDIX C: FIRST ORDER TRANSITION IN NONSYMMETRIC DYNAMICS ON DEGREE REGULAR RANDOM GRAPHS}

To obtain the PDFs in Fig. 12, a relatively small system size ( $n=59$ nodes) was chosen. The dynamics was simulated until a sufficient number of transitions between the extremal states had taken place, to be able to obtain an approximation of the statistical occupation of the different values $w-u$. For larger system sizes the transitions were very rare or did not occur at all during the computation. We, however, checked smaller system sizes and found compatible results.

\section{APPENDIX D: POLARIZATION IN TREE APPROXIMATION}

For the tree approximation, which was discussed in Sec. $\mathrm{V}$, for star topologies with several numbers of sites we computed the occupation probabilities for all star states, both
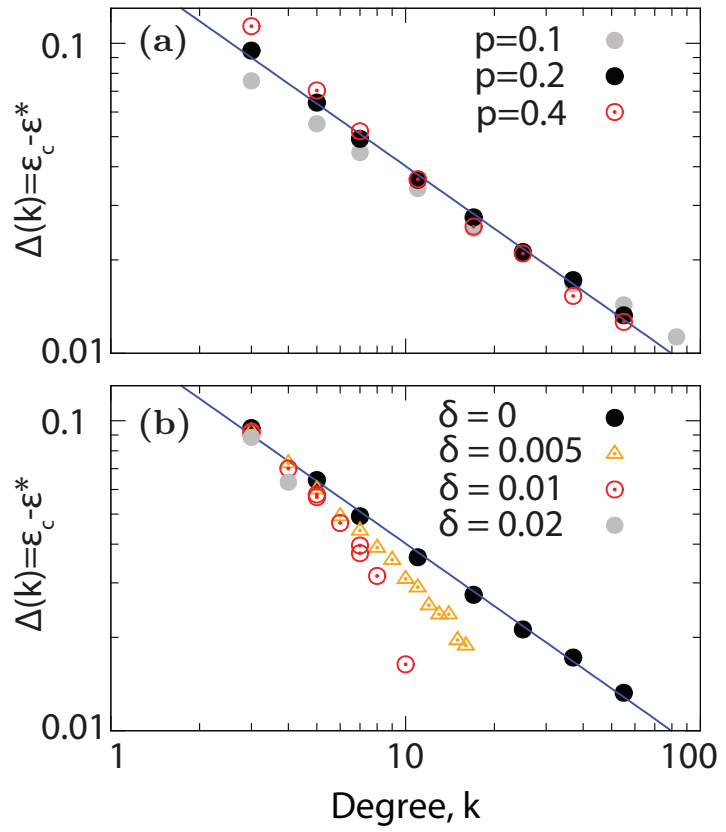

FIG. 14. Transition point vs degree and noise bias. (a) Difference of fully connected, infinite system bifurcation point $\varepsilon_{c}$ and regular graph transition point $\varepsilon^{*}$ for varying values of degree $k$ and different values of $p$. System sizes: 8000 for $p=0.1$ and $p=0.4,4000$ for $p=0.2$. (b) System with noise bias, i.e., for $u \rightarrow v$ and $v \rightarrow w$ noisy transitions were increased to $\varepsilon+\delta$ while the opposite direction was decreased to $\varepsilon-\delta$. Values of $\delta$ as indicated in plot. System sizes: $N=5000$, except for $\delta=0$, where $N=5000$ was used. 
for the isolated star (red links in Fig. 6) as well as the tree approximation (all links in Fig. 6), where the star is embedded in a Bethe lattice. Weighting by their relative occupation probabilities, the self-consistent star states were then binned by their respective values of $w-u$. For the resulting distribution function of $w-u$ we extracted the local probability maxima. We then repeated this analysis for many values of $\varepsilon$. The resulting pattern of maxima (Fig. 13) shows transitions from bimodal to unimodal statistics.

\section{APPENDIX E: DYNAMICS ON DEGREE REGULAR NETWORKS WITH $\langle k\rangle>3$}

For fixed $p<1$, we monitored the abrupt transition from bistability to monostability in degree-regular networks of increasing $\langle k\rangle$. Over approximately two orders of magnitude of $\langle k\rangle$ such transitions are always found (Fig. 14). As $\langle k\rangle \rightarrow N$, the transition point $\varepsilon^{*}(p)$ approaches the bifurcation point $\varepsilon_{c}(p)$ of the fully connected system. We find the difference $\Delta(k) \equiv\left|\varepsilon_{c}(p)-\varepsilon^{*}(p)\right|$ to decrease algebraically as $\mid \varepsilon_{c}(p)-$ $\varepsilon^{*}(p) \mid \sim k^{-s(p)}$ with $0<s(p)<1$ weakly increasing with the asymmetry parameter $p$. The algebraic dependence of $\Delta(k)$ on degree can be removed by a noise bias, where $\varepsilon$ is replaced by $\varepsilon+\delta(\varepsilon-\delta)$ for transitions towards $w(u)$. Such noise bias is found to give a specific degree cutoff, where $\varepsilon_{c}=\varepsilon^{*}$ is met. It is important to note that for sufficiently small degree, even under the action of a noise bias, a substantial offset $\Delta(k)$ remains. This means that our results are not limited to the special case of unbiased (neutral) noise but are valid for a larger class of models.
[1] J. E. Ferrell, Curr. Biol. 22, R458 (2012).

[2] J. O. Haerter, C. Lövkvist, I. B. Dodd, and K. Sneppen, Nucleic Acids Res. 42, 2235 (2014).

[3] B. Skyrms, The Stag Hunt and the Evolution of Social Structure (Cambridge University Press, Cambridge, 2004).

[4] A. Bar-Even, J. Paulsson, N. Maheshri, M. Carmi, E. O’Shea, Y. Pilpel, and N. Barkai, Nat. Genet. 38, 636 (2006).

[5] J. Fernández-Gracia, K. Suchecki, J. J. Ramasco, M. San Miguel, and V. M. Eguíluz, Phys. Rev. Lett. 112, 158701 (2014).

[6] R. I. Dunbar, J. Human Evol. 22, 469 (1992).

[7] Y. Togashi and K. Kaneko, Phys. Rev. Lett. 86, 2459 (2001).

[8] A. Kirman, Q. J. Econ. 108, 137 (1993).

[9] K. Suchecki and J. A. Hołyst, Phys. Rev. E 80, 031110 (2009).

[10] Y. Ye, L. Wang, and N. Xie, PloS One 8, e67924 (2013).

[11] G. García-Pérez, M. Boguñá, and M. Á. Serrano, Sci. Rep. 5, 9714 (2015).

[12] G. Mosquera-Donate and M. Boguñá, Phys. Rev. E 91, 052804 (2015).

[13] A. Carro, R. Toral, and M. San Miguel, Sci. Rep. 6, 24775 (2016).

[14] H. Wio and K. Lindenberg, in Proceedings of the AIP Conference, Vol. 658 (AIP, New York, 2003), pp. 1-62.
[15] T. Çağatay, M. Turcotte, M. B. Elowitz, J. Garcia-Ojalvo, and G. M. Süel, Cell 139, 512 (2009).

[16] D. Abbott, B. R. Davis, and J. Parrondo, in AIP Conference Proceedings (Institute of Physics Publishing, New York, 1999), pp. 213-220.

[17] G. P. Harmer and D. Abbott, Stat. Sci. 14, 206 (1999).

[18] J. M. R. Parrondo, G. P. Harmer, and D. Abbott, Phys. Rev. Lett. 85, 5226 (2000).

[19] R. Toral, Fluctuation Noise Lett. 1, L7 (2001).

[20] D. Abbott, Fluctuation Noise Lett. 9, 129 (2010).

[21] I. B. Dodd, M. A. Micheelsen, K. Sneppen, and G. Thon, Cell 129, 813 (2007).

[22] C. Lövkvist, I. B. Dodd, K. Sneppen, and J. O. Haerter, Nucleic Acids Res. 44, 5123 (2016).

[23] B. L. Chen, D. H. Hall, and D. B. Chklovskii, Proc. Nat. Acad. Sci. USA 103, 4723 (2006).

[24] D. J. Watts and S. H. Strogatz, Nature (London) 393, 440 (1998).

[25] V. Sood and S. Redner, Phys. Rev. Lett. 94, 178701 (2005).

[26] B. Gonçalves, N. Perra, and A. Vespignani, PloS One 6, e22656 (2011).

[27] J. O. Haerter, B. Jamtveit, and J. Mathiesen, Phys. Rev. Lett. 109, 168701 (2012). 